\title{
Characterization of the Weld Regions within Duplex Stainless Steels using Magnetic Force Microscopy
}

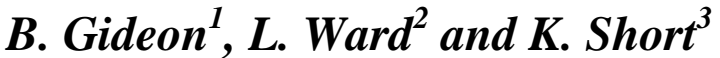

\author{
${ }^{1}$ ARV Offshore, Bangkok, Thailand \\ ${ }^{2}$ School of Civil, Environmental and Chemical Engineering, RMIT University, GPO \\ Box 2476V, Melbourne, Vic. 3001, Australia \\ ${ }^{3}$ Australian Nuclear Science and Technology Organization, Lucas Heights, NSW, 2234, \\ Australia
}

\begin{abstract}
Standard metallography and optical microscopy are well established techniques for the characterization of duplex stainless steels (DSS), which consist of approximately $50 \%$ ferrite and 50\% austenite. Recently, the use of atomic and magnetic force microscopies (AFM and MFM respectively) have been employed to differentiate between magnetic and non magnetic phases in materials. Such techniques would be valuable to identify different phases in duplex stainless steels, particularly the weld regions, and would thus compliment standard metallographic and optical microscopy techniques. In particular, AFM and MFM would be particularly valuable for identification of phases within the different weld regions (root, fill and cap).

In the present study, Gas Tungsten Arc Welded (GTAW) DSS samples, as a function of heat input and weld configuration, were subject to standard metallographic practices (ferrite content determination, Vickers hardness measurements, Charpy impact studies and transverse tensile testing) in addition to MFM analysis. The metallographic tests revealed that the weld properties were acceptable in accordance with current industrial standards. The MFM results of the weld metal shows the formation of both a finer and coarse structure within the weld metal, which is dependent on the level of undercooling.
\end{abstract}

Key Words: GTAW Welding, Duplex Stainless Steels, Mechanical Properties, Magnetic Force Microscopy, Characterization 


\section{INTRODUCTION}

Traditionally, the common methods for studying the microstructure of duplex stainless steels (DSS), in particular the weld regions, have been quantitative metallography and microhardness techniques. However, a technique has been developed to compliment these conventional mehods, utilizing a scanning probe microscope in magnetic imaging mode, known as magnetic force microscopy (MFM), which enables the ferrite regions to be distinguished from the austenite regions, using their magnetic characteristics. The ferrite regions are ferromagnetic, in contrast to the austenite regions, which are paramagnetic [1]. The spatial variation of the magnetic force interaction between these regions can be studied using MFM and is now recognized as a powerful tool for the characterization of Duplex Stainless Steels (DSS) [2, 3].

MFM imaging mode is based on non-contact Atomic Force Microscopy (AFM), with the tip modulated at or near its resonant frequency by means of a piezoelectric element and the cantilever coated with a magnetic material. When resonated over the sample surface, the tip-sample interaction includes both surface and magnetic forces. A limitation of this technique is the ability to accurately align the information obtained on surface topography characteristics using the scanning probe microscopy in atomic force microscopy (AFM) mode, with information obtained on the magnetic contrast of differently magnetized domains using the scanning probe microscope in MFM mode. However, these problems have been overcome by adopting a two-pass procedure, whereby a second signal is measured in addition to AFM surface topography. For MFM measurements, this is possible by using a CoCr-coated tip. This set-up allows for a very easy combination of the two techniques by simply changing some software parameters. Consequently, this specific procedure has been adopted in the current investigation. Previous studies by Takaya et al [4] on the application of MFM for studying $\mathrm{Cr}$ depleted regions of 304 stainless steel showed a strong correlation between the depleted regions and the degree of sensitization to stress corrosion cracking. AFM and MFM studies by Dias and Andrade [5] showed that the clarity of magnetic patterns was strongly dependant on the type of magnetic tip employed and the tip - surface separation distance.

In the present investigation, MFM studies were carried out on the weld and parent metal regions of four duplex stainless steel weld samples, in order to compliment information provided by conventional metallography and microscopy techniques, for structural and 
morphological characterization of the DSS welds.

\section{THEORY OF MAGNETIC FORCE MICROSCOPY FOR IMAGING DUPLEX STAINLESS STEELS}

In MFM, the magnetic fields adjacent to a sample are detected with sub-micron resolution, by scanning a magnetic probe over the surface and recording the changes in its phase or resonant frequency [6]. Once set in place in the instrument, the tip is oscillated at its resonant frequency by a piezoelectric element, and scanned over the sample surface. The topography of the sample surface is obtained in the first pass by lightly tapping the surface with the tip. In the second pass, the tip is lifted off the surface by a predetermined distance (in this study, between 50 and $100 \mathrm{~nm}$ ) so that only the magnetic forces affect the tip, thus avoiding interference from the surface topography $[6,7]$. The tip is then scanned along the same line following the topographic surface contour recorded during the first pass, so that the tip-sample distance, and hence the resolution, are maintained constant. In this way, the phase shift induced by the magnetic force gradient between the tip and the sample can be recorded, yielding an image of the magnetic patterns over the surface, which in the case of DSS can be associated to the microstructure of the sample (Fig. 1).

Fig. 1 Schematic diagram showing the principle of MFM imaging for DSS Welds Phase shifts $(\Delta \theta)$ between oscillations of the cantilever and the piezoelectric actuators measured by equation 1 for small amplitudes cantilever as follows:

$\Delta \theta=\frac{Q}{k} F^{\prime}$

Equation 1

$\mathrm{Q}$ is the free oscillation quality factor $(\approx 165 \pm 10$ in air $), \mathrm{k}$ is the spring constant $(\approx 3 \mathrm{~N} / \mathrm{m})$ and $\mathrm{F}^{\prime}$ is the vertical gradient of the magnetic force on the tip of the cantilever.

Therefore, F' can be calculated as shown in equation 2 :

$F^{\prime}(r)=\frac{\partial F}{\partial z}=\int_{t i p} \frac{\partial^{2}}{\partial z^{2}}\left\{M^{t i p}\left(r^{\prime}\right) \cdot H^{\text {sample }}\left(r+r^{\prime}\right)\right\} d V^{\prime}$--------------------Equation 2

$M^{\text {tip }}\left(r^{\prime}\right)$ is the magnetization of the volume element in the tip and $H^{\text {sample }}\left(r+r^{\prime}\right)$ is the stray field from the sample [8]. The liftoff between the tip and the sample surface is about $100 \mathrm{~nm}$. Specimens were polished mechanically before the MFM observation.

\section{EXPERIMENTAL}

\subsection{Welding of Duplex Stainless Steels}


The parent material chosen for the investigation was a $10 \mathrm{~mm}$ wall thickness, $250 \mathrm{~mm}$ diameter DSS linepipe corresponding to UNS 31803 specifications. The filler material used was the conventional ER2209 AWS A5.9-93 classification. Full details of the chemical composition of both the parent material and filler material are listed in Table 1 , confirming that the primary solidification mode was ferrite.

Table 1. Chemical composition of DSS pipe and filler material.

\begin{tabular}{|l|l|l|l|l|l|l|l|l|l|l|l|l|l|l|}
\hline Pipe & & $\mathrm{C}$ & $\mathrm{Mn}$ & $\mathrm{P}$ & $\mathrm{S}$ & $\mathrm{Si}$ & $\mathrm{Ni}$ & $\mathrm{Cr}$ & $\mathrm{Mo}$ & $\mathrm{N}$ & $\mathrm{Cu}$ & $\mathrm{Pren}$ & $\mathrm{Cr}_{\mathrm{eq}}$ & $\mathrm{Ni}_{\mathrm{eq}}$ \\
\cline { 2 - 14 } & Min & - & - & - & - & - & 5.00 & 21.50 & 3.00 & 0.15 & - & 35 & - & - \\
\cline { 2 - 13 } & Max & 0.030 & 2.0 & 0.025 & 0.015 & 1.0 & 6.50 & 23.00 & 5.50 & 0.20 & 0.16 & - & 32.04 & 10.78 \\
\hline $\begin{array}{l}\text { Filler } \\
\text { Materia } \\
1\end{array}$ & Max & 0.016 & 1.69 & - & - & 0.42 & 8.60 & 23.07 & 3.20 & 0.160 & 0.16 & $\geq 35$ & 28.09 & 11.90 \\
\hline
\end{tabular}

Note; $\mathrm{Cr}_{\mathrm{eq}}=\mathrm{Cr}+1.37 \mathrm{Mo}+1.5 \mathrm{Si}+2 \mathrm{Nb}+3 \mathrm{Ti}$ and $\mathrm{Ni}_{\mathrm{eg}}=\mathrm{Ni}+22 \mathrm{C}+0.31 \mathrm{Mn}+14.2 \mathrm{~N}+\mathrm{Cu}$

Welding was performed using the manual Gas Tungsten Arc Welding (GTAW) technique. Two different join configurations were adopted, namely double bevel single $\mathrm{V}$ bevel and double bevel single $\mathrm{U}$ joint configuration. Full details of the weld parameters, to include number of passes, arc travel speed and heat input, are given in Table 2. For the $\mathrm{V}$ bevel configuration, welding was performed at low $(1515.38 \mathrm{~J} / \mathrm{min}$ average) and high (2000.97 J/min average) heat input powers. For the $\mathrm{U}$ groove configuration, two welding operations were performed at similar heat input powers (1429.35 J/min). These will be referred to as weld conditions 1 to 4 respectively.

Upon completion of welding, all test conditions were visually inspected for surface defects both internally and externally. Liquid dye penetrant tests were performed 4 hours after completion of welding, and radiography (X-ray) was performed 24 hours later to determine the integrity of the girth welds.

Table 2. Weld conditions for 4 DSS samples used in this study. 


\begin{tabular}{|c|c|c|c|}
\hline & & $\mathrm{mm} / \mathrm{min}$ & $\mathrm{J} / \mathrm{min}$ \\
\hline & 1 (weld root) & 51.00 & 1474.71 \\
\hline \multirow{2}{*}{ Condition 1} & 2 (weld fill) & 123.00 & 883.12 \\
\hline & 3 (weld fill) & 66.00 & 1745.45 \\
\hline \multirow[t]{3}{*}{ V groove } & 4 (weld fill) & 64.00 & 1788.00 \\
\hline & 5 (weld cap) & 64.00 & 1685.63 \\
\hline & & Average & 1515.38 \\
\hline \multirow{2}{*}{ Condition 2} & 1 (weld root) & 45.00 & 1591.20 \\
\hline & 2 (weld fill) & 105.00 & 1440.46 \\
\hline \multirow{4}{*}{ V groove } & 3 (weld fill) & 79.00 & 2756.05 \\
\hline & 4 (weld cap) & 94.00 & 2216.17 \\
\hline & & Average & 2000.97 \\
\hline & 1 (weld root) & 110.00 & 419.78 \\
\hline \multirow{2}{*}{ Condition 3} & 2 (weld fill) & 62.00 & 757.55 \\
\hline & 3 (weld fill) & 38.00 & 1733.05 \\
\hline \multirow[t]{3}{*}{ U groove } & 4 (weld fill) & 40.00 & 2194.80 \\
\hline & 5 (weld cap) & 43.00 & 2041.67 \\
\hline & & Average & 1429.37 \\
\hline \multirow{2}{*}{ Condition 4} & 1 (weld root) & 115.00 & 420.31 \\
\hline & 2 (weld fill) & 66.00 & 942.55 \\
\hline \multirow{3}{*}{ U groove } & 3 (weld fill) & 73.00 & 2219.18 \\
\hline & 4 (weld cap) & 57.00 & 2135.37 \\
\hline & & Average & 1429.35 \\
\hline
\end{tabular}

\subsection{Mechanical Testing of Welded Duplex Stainless Steels}

Ferrite contents of the four weld conditions were measured using both the MagnaGauge and Fischer ferrite-scope methods and, as a comparison, determined metallographically by the point count method [9].

Vickers hardness measurements were made with a $10 \mathrm{~kg}$ load in the parent material, heat affected zone (HAZ), weld cap, weld fill and weld root regions. Transverse tensile test specimens were used to determine the tensile values whilst the more restrictive 
transverse side bend specimens were used in lieu of the root and face bend tests, commonly adopted in accordance with weld procedure qualifications. Charpy impact tests were performed to assess the notch toughness of samples extracted from the weldments, in accordance with ASTM A 370 standards [10].

\subsection{Magnetic Force Microscopy Studies of Welded Duplex Stainless Steels}

MFM studies were conducted on metallographically prepared cross-sections of the welds, after grinding and polishing using $\mathrm{SiC}$ abrasive papers and diamond paste. The scanning probe microscopy (Digital Instruments), operating in tapping and lift modes was employed to study the topographic and magnetic features of the DSS samples. Topographic and magnetic force data were taken in the same scan. In order to produce reliable images, repeated scans in different directions were done to ensure reproducibility of the features. Various scan sizes and speeds were tested to enhance height and magnetic induced signals, thus minimizing tip hysteresis and the delay between line scans.

A cantilever equipped with a special coated tip was scanned over the surface of the DSS sample, using tapping mode in order to obtain surface topography profiles. To obtain magnetic images, lift mode was adopted, whereby the tip was then raised just above the sample surface. The surface topography was then scanned while being simultaneously monitored for the influence of magnetic forces. These influences were measured using the principle of force gradient detection $[8,11,12]$. In the absence of magnetic forces, the cantilever has a resonant frequency that is shifted by an amount proportional to vertical gradients in the magnetic forces on the tip. In the present work, the frequency modulation detection method was used to measure the resonance frequency shift. The cantilever is maintained by a feedback loop using the signal from the deflection sensor; thus, changes in the oscillation frequency caused by variations of the force gradient of the tip-sample interaction are directly measured [13].

\section{Results and Discussion}

\subsection{Mechanical Properties of Welded Duplex Stainless Steels}

Vickers hardness testing revealed that for all the four fusion zones, hardness values in the range of 235-285 $\mathrm{HV}_{10}$ were exhibited. A summary of the results of the mechanical properties of the welded duplex stainless steels is given in Table 3. It can be seen that 
the notch toughness values obtained by Charpy impact testing at $-43{ }^{\circ} \mathrm{C}$ show that, although slight differences exist in the ferrite content among the four test conditions, condition 1 and 2 had impact values of 116 joules while condition 3 and 4 have impact energy values of 97 joules and 100 joules, indicating a reduction of $16.4 \%$ and $13.8 \%$ respectively. This suggests that the differences in the toughness cannot be explained simply in terms of the changes in ferrite content. The fact that all four weld conditions retain much of their toughness even at $-43{ }^{\circ} \mathrm{C}$ may not be due to a reduction in ferriteaustenite ratio alone. Tensile strength values for all 4 conditions were within the range of $774 \mathrm{~N} / \mathrm{mm}^{2}$ to $794 \mathrm{~N} / \mathrm{mm}^{2}$. In summary, the metallographic tests revealed that the weld properties were acceptable in accordance with current industrial standards.

Table 3 Summary of the results of the mechanical properties of the welded duplex stainless steels

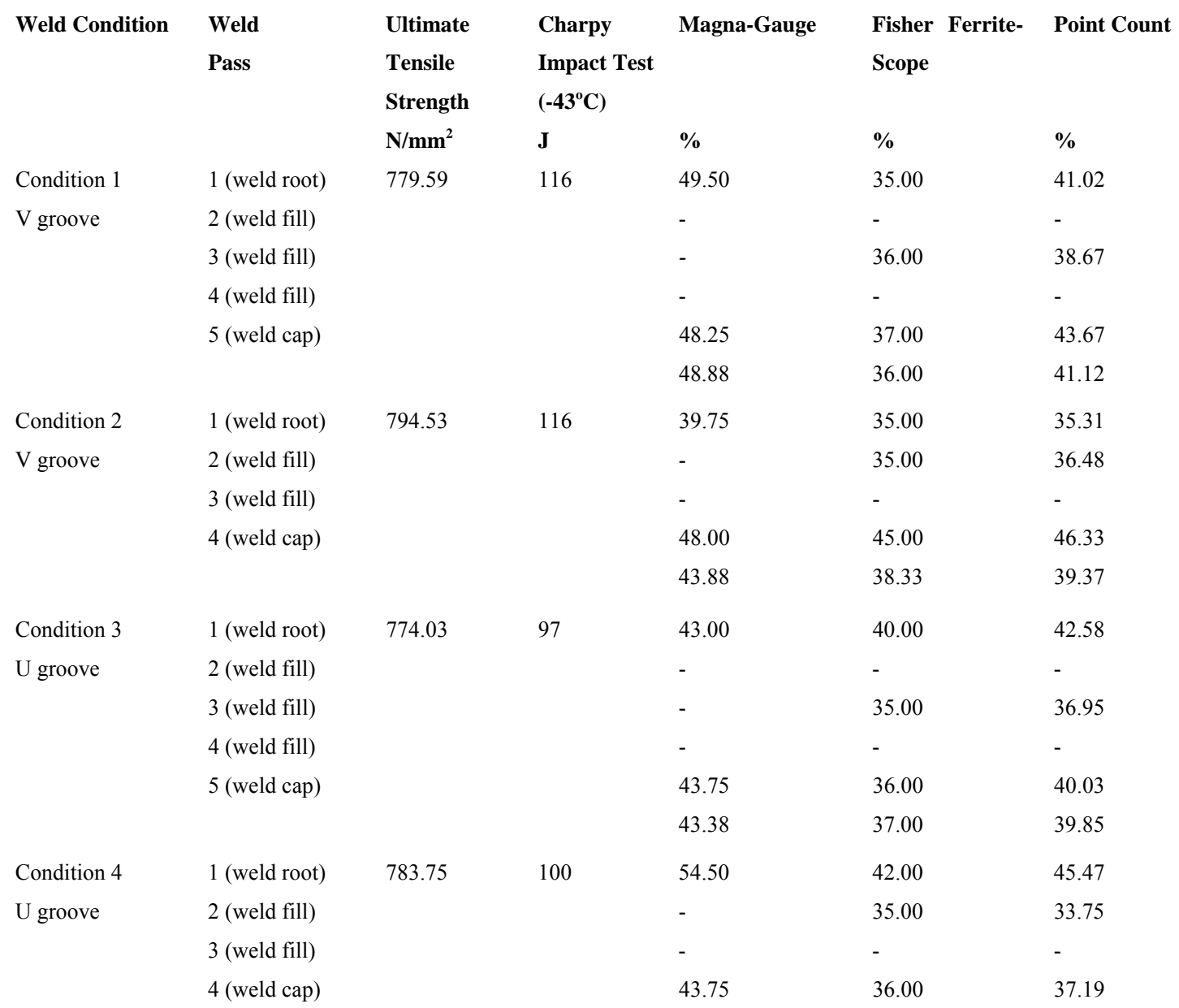




\subsection{Magnetic Force Microscopy Studies of Welded Duplex Stainless Steels}

Topographic images and corresponding magnetic force images for all weld conditions (conditions 1 to 4) are shown in Figs. 2 - 5 respectively. Each figure consists of MFM images taken from three regions of the weld, namely the root, fill and cap, in addition to the AFM image. In general, the topographic images, although showing the surface topography of the metallographically prepared weld, reveal no information about the phases present. However, two distinct regions are easily identified in the MFM images. The lighter regions represent the paramagnetic austenite regions, while the striated, darker regions represent the ferromagnetic ferrite regions. Variations in the relative distributions of the austenite and ferrite regions can be observed not only in the different weld areas for any given configuration, but also between specific weld regions over all configurations examined.

For weld condition 1 (V configuration, low heat input), the root and fill regions of the weld consist mainly of austenite in the forms of large elongated platelets and small fine, eqiaxed dendritic regions (Figs. 2 (a) and 2 (b)), surrounded by fine discontinuous regions of ferrite. In contrast the cap region, as shown in Fig. 2 (c), show larger continuous regions of ferrite, in addition to a long continuous band of austenite forming at the grain boundary. For the same configuration with higher heat input (Fig. 3), similar observations were evident in all three regions, compared with the lower heat input.

For weld conditions 3 and 4 (Figs. 4 and 5 respectively), using the U configuration at low heat input, similar structures were observed compared with conditions 1 and 2 . However, large regions of ferrite were observed in the fill regions of the weld for condition 4 (Fig. 5 (b)). Further, the root region for condition 3, as shown in Fig. 4 (c), consisted of elongated bands of austenite surrounded by ferrite. Such a structure is typical of the parent metal structure, which would have elongated austenite grains embedded in a ferrite matrix.

The results of the MFM imaging in general, suggest that the austenite regions observed 
in the DSS weld metal regions are formed from ferrite in three modes, viz., as allotriomorphs at the prior-ferrite grain boundaries, as Widmanstätten side-plates growing into the grains from these allotriomorphs and as intragranular precipitates. In the micrographs, the grain boundary allotriomorphs and Widmanstätten austenite are clearly seen. However, the austenite seen within the grain could be either intragranular precipitates or Widmanstätten austenite intercepted transverse to the long axis. The findings from the MFM work conducted in this study here confirm the findings from a previous study [14] which looked at the structure of these duplex stainless steels using conventional microscopy.

The different austenite / ferrite microstructures observed in the weld regions, such as the presence of discontinuous grain boundary austenite layers (Figs. 2(a) and 2 (b)), Widmanstätten austenite side-plates, austenite intragranular precipitates and intragranular acicular ferrite are thought to be associated with, and hence explained in terms of variations in transformation rates and the degree of undercooling [15].

The formation of grain boundary and side-plate fractions require a relatively smaller driving force [16] and therefore can occur at higher temperatures with little undercooling. The formation of intragranular acicular ferrite, on the other hand, requires a greater degree of undercooling and therefore occurs at lower transformation temperatures. It is likely that a similar transformation sequence is adopted during the microstructural evolution of the regions associated with the DSS weld. Thus the grain boundary austenite and Widmanstätten side-plates form early at higher temperatures, while the intragranular austenite particles require a greater driving force and precipitate later at a lower temperature. This can also be seen in the MFM images, where the intragranular precipitates are seen to form in the regions partitioned by the Widmanstätten plates. When cooling occurs rapidly in the cap region of the welds, it is expected that the transformation product requiring a higher degree of undercooling is formed, and hence the greater volume of ferrite observed in the cap region compared with the root and fill regions.

The MFM technique was capable of clearly imaging the magnetic domain structure of the ferrite phase that surrounds the "islands" of austenite, which appear flat and uniform due to their paramagnetic properties. Clear bands of ferrite could be easily distinguished, but a closer examination of the images revealed other regions, considered to be ferrite, that did not exhibit the more typical striped magnetic domain configuration 
associated with ferrite. The different appearances of the magnetic domains in the ferrite phase can be explained in terms of the orientation of the magnetic domains.

One of the most important factors in MFM imaging is the actual orientation of the magnetic domains of the sample, which, in turn, depends on the crystallographic orientation of the ferrite. Therefore, it should be expected that ferrite grains with different crystallographic orientations yield different magnetic patterns in the MFM images. Other factors that can affect the contrast in the MFM images are the geometry of the magnetic domains, and the fact that even domains that are underneath the surface (i.e. non superficial) can be detected, which may render different contrast than the superficial ones. A further drawback from the use of this technique is the ability to resolve features associated with the magnetic domains and the possible inclusion of fine precipitates such as secondary austenite, $\chi$ and $\sigma$ phases.

\section{CONCLUSIONS}

1. The metallagraphic tests carried out on the different weld regions, revealed that the weld properties were acceptable in accordance with current industrial standards

2. Magnetic force microscopy was successfully conducted on the various regions of the duplex stainless steel welds using the scanning probe microscope in both lift and tapping mode.

3. The magnetic force microscopy images revealed (i) the formation of both fine and coarse structure within the weld metal and (ii) clearly defined austenite and ferrite regions within the different weld passes (root, fill, cap) for the different weld configurations adopted in the study here.

4. The different austenite / ferrite microstructures observed in the weld regions, such as the presence of discontinuous grain boundary austenite layers, Widmanstätten austenite side-plates, austenite intragranular precipitates and intragranular acicular ferrite are thought to be associated with, and hence explained in terms of variations in transformation rates and the degree of undercooling

5. MFM is a powerful tool to use for differentiating the austenite and ferrite phase in duplex stainless steels. 


\section{ACKNOWLEDGEMENTS}

The authors would like to thank Dr. Lim Chiang Liang of Metacos for preparing the duplex stainless steel samples for metallographic studies conducted. The authors would like to acknowledge support provided by the Australian Institute of Nuclear Science and Engineering (AINSE - grant no. AINGRA06184P), to allow the magnetic force microscopy studies to be conducted at the Australian Nuclear Science and Technology Organization (ANSTO). Acknowledgement goes to ARV Offshore for their continuing support towards this research program.

\section{REFERENCES}

[1] D. Peckner, I.M. Bernstein, in: H.B. Crawford, B. Gatewood (Eds. ), Handbook of Stainless Steels, McGraw-Hill, Caledonia, 1977.

[2] M.S. Andrade, J.M.R Vilela, A.C.C. Reis, J.A. Sluss, V.T.L. Buono, Acta Microsc. 5B (1996) 266.

[3] B.R.A. Neves, M.S. Andrade, Appl. Phys. Lett. 74 (1999) 2090.

[4] S. Takaya, T. Suzuki, Y. Matsumoto, K. Demachi and M. Uesaka, J. Nuclear Materials 327 (2004) 19-26

[5] A. Dias and M.S. Andrade, Appplied Surface Science 161 (2000) 109-114

[6] J. Wittborn, Nanoscale Studies of Functional Materials using Scanning Probe Microscopy, Doctoral Thesis. Royal Institute of Technology, Stockholm, Sweden, p. 10 (2000).

[7] B. R. A. Neves and M. S. Andrade, Identification of two Patterns in Magnetic Force Microscopy of Shape Memory Alloys, Appl. Phys. Lett., 74, 2090 (1999).

[8] R. Wiesendanger, Scanning Probe Microscopy and Spectroscopy - Methods and Applications, Cambridge Univ. Press, Cambridge, 1996.

[9] ASTM E562, Standard Test Method for Determining Volume Fraction by Systematic Manual Point Count

[10] ASTM A370, Standard Test Methods and Definitions for Mechanical Testing of Steel Products

[11] J.L. Hunter, J. Bechhoefer, J. Vac. Sci. Technol., B 12 (1994 ) 2251.

[12] D. Sarid, in: M. Lapp, H. Stark (Eds. ), Scanning Force Microscopy, Oxford Univ. Press, New York, 1991.

[13] C. Schonenberger, S.F. Alvarado, S.E. Lambert, I.L. Sanders, J. Appl. Phys. 67 (1990) 7278 . 
[14] Gideon B, Ward L P and Biddle G, Metallurgical Characterisation of Duplex Stainless Steel and their Susceptibility to Intergranular Corrosion, Proc. European Corrosion Federation (EUROCORR 2006) Conference Maastricht, Netherlands, 24 28 Sep (2006) Session K, pp. 190-191

[15] L. Karlsson: 'Intermetallic Phase Precipitation in Duplex Stainless Steels and Weld Metals: Metallurgy, Influence on Properties and Testing Aspects', Welding in the World, vol. 43, no. 5, 1999.

[16] B.J. Ginn and T.G. Gooch: 'Effect of Intermetallic Content on Pitting Resistance of Ferritic-Austenitic Stainless Steels', proc. conf. Stainless Steels'91 Science and market, Chia Laguna Sardinia, Italy, 1999, vol. 3, p. 81-89 